\title{
PIERCING A DISK ALONG A CELLULAR SET
}

\author{
D. R. MCMILLAN, JR. ${ }^{1}$
}

1. Introduction. Let $S$ be a 2 -sphere topologically embedded in the 3 -sphere $S^{3}$. A point $p \in S$ is called a piercing point of $S$ if $S$ can be pierced with a tame arc at $p$. In [6] Gillman improved some earlier results of $\mathrm{R}$. H. Bing on the existence of piercing points to show that the nonpiercing points of $S$ form a 0 -dimensional $F_{\sigma}$ subset of $S$. In this note, we show (Theorem 2) that no subset of $S$ which is cellular in $S^{3}$ can contain more than one nonpiercing point of $S$. As a corollary (Theorem 3 ), we find that if both complementary domains of $S$ are open 3 -cells, then at most two points of $S$ are nonpiercing points.

The proof of Theorem 2 depends on Theorem 1, which says that if a cellular set "topologically collapses" to a subset, then the subset is also cellular. For example, if a topological 2-cell $D$ is cellular in $S^{3}$ then any topological 2-cell in Int $D$ is also cellular in $S^{3}$. Another important tool in the proof is a theorem of Gillman and Martin (stated in [11] as Lemma 4) to the effect that a cellular arc in $S^{3}$ cannot fail to be locally tame only at its endpoints.

If $K$ is a finite simplicial complex and $L$ is a subcomplex of $K$, the statement that $K$ contracts (or "collapses") to $L$ is understood in the sense of J. H. C. Whitehead [14]. A crumpled cube $K$ is any space homeomorphic to the closure of a complementary domain of a 2sphere $S$ in $S^{3}$. We write $S=\mathrm{Bd} K$. If $K$ is a crumpled cube and $S=\operatorname{Bd} K$, then $p \in S$ is said to be a piercing point of $K$ if there exists an embedding $h: K \rightarrow S^{3}$ such that $h(S)$ can be pierced with a tame arc at $h(p)$. In [8] and [9] it was shown that for any crumpled cube $K$ there exists an embedding $g: K \rightarrow S^{3}$ such that the closure of $S^{3}-g(K)$ is a 3-cell; and in [11] it was shown that $p$ is a piercing point of $K$ if and only if $g(\mathrm{Bd} K)$ can be pierced with a tame arc at $g(p)$. Further, in [13] it was noted that if $S$ is a topological 2-sphere in $S^{3}$ then $p \in S$ is a piercing point of $S$ if and only if $p$ is a piercing point of both of the crumpled cubes bounded by $S$.

We use $\Delta^{n}(n \geqq 0)$ to denote a closed $n$-simplex. A topological space $X$ is called a compact absolute retract if for some $n$, it can be embedded in $\Delta^{n}$ as a retract of $\Delta^{n}$. If $X \subset$ Int $M^{n}$ is a compact absolute retract and $M^{n}$ is a piecewise-linear $n$-manifold, we say that $X$ satisfies the cellularity criterion if: For each open set $U$ such that $X \subset U \subset M^{n}$, there exists an open set $V \subset M^{n}$ such that $X \subset V \subset U$ and such that

Received by the editors December 12, 1966.

1 Alfred P. Sloan Fellow. 
each loop in $V-X$ is contractible in $U-X$. In [12], it was shown that if $n \geqq 5$ or if $n=3$ and $M^{3}=S^{3}$, then $X$ is cellular in $M^{n}$ if and only if $X$ satisfies the cellularity criterion.

2. Topological collapsing. The following is a generalization of the theorem that "each subarc of a cellular arc is cellular" (see Theorem 6 of [12]).

THEOREM 1. Let $K$ be a contractible, finite simplicial complex, and $L$ a subcomplex of $K$ such that $K$ contracts to $L$. Let $h: K \rightarrow \operatorname{Int} M^{n}$ be a topological embedding, where $M^{n}$ is a piecewise-linear n-manifold, and $n \geqq 3$. Then, if $h(K)$ satisfies the cellularity criterion, so also does $h(L)$. Hence, if $n \neq 4$ and if $h(K)$ is cellular, so also is $h(L)$.

Proof. The last assertion follows from the first part of the conclusion and from Theorems 1 and $1^{\prime}$ of [12]. It remains only to show that if $L$ is obtained from $K$ by one elementary contraction and if $h(K)$ satisfies the cellularity criterion, then $h(L)$ does also. We assume that $n \geqq 3$.

To do this, let $U \subset$ Int $M^{n}$ be an open set containing $h(L)$. By hypothesis, there is a principal simplex $s^{k}(k \leqq n)$ of $K$, and a face $t^{k-1}$ of $s^{k}$ such that $t^{k-1}$ is a proper face of no other simplex of $K$, and

$$
L=K-\left(\operatorname{Int} s^{k}\right)-\left(\operatorname{Int} t^{k-1}\right) .
$$

Suppose $v$ is the vertex of $s^{k}$ opposite $t^{k-1}$, and let $\pi: s^{k}-v \rightarrow t^{k-1}$ be the radial projection map. Let $u^{k-1}$ be a simplex in Int $t^{k-1}$ such that $\mathrm{Bd} u^{k-1}$ is concentric with $\mathrm{Bd} t^{k-1}$ and such that

$$
\pi\left(s^{k}-h^{-1}(U)\right) \subset \text { Int } u^{k-1} \text {. }
$$

Choose a point $p$ on the interior of the line segment joining $v$ to the barycenter of $t^{k-1}$, and so close to $v$ that the join of $p$ and $\mathrm{Bd} u^{k-1}$ is a $(k-1)$-cell $C^{k-1}$ separating $L$ and $s^{k}-h^{-1}(U)$ in $K$. (If $k=1$, we take $u^{k-1}=t^{k-1}$ and $C^{k-1}=p$.) Let $B^{k}$ be the join of $p$ and $u^{k-1}$.

Let $W$ be an open set in $M^{n}$ such that

$$
h\left(C^{k-1}\right) \subset W \subset \bar{W} \subset U-h(L)
$$

and such that $\bar{W}$ is contractible to a point in $U-h(L)$. This last condition is possible because $h\left(C^{k-1}\right)$ is a compact absolute retract. Let $Q$ be an open set in $M^{n}$ such that

$$
h(L) \cup h\left(\overline{s^{k}-B^{k}}\right)-W \subset Q \subset \bar{Q} \subset U-h\left(B^{k}\right) .
$$

Then $U^{*}=Q \cup W \cup\left(M^{n}-\bar{Q}\right)$ is an open set in $M^{n}$ containing $h(K)$. Since $h(K)$ satisfies the cellularity criterion, there is an open set $V^{*}$ such that $h(K) \subset V^{*} \subset U^{*}$ and each loop in $V^{*}-h(K)$ is contractible 
in $U^{*}-h(K)$. Let $V$ be the component of $V^{*}-\bar{W}$ containing $h(L)$. Then $V \subset Q-\bar{W} \subset U$.

We claim that each loop in $V-h(L)$ is contractible in $U-h(L)$. Let a loop

$$
f: \mathrm{Bd} \Delta^{2} \rightarrow V-h(L)
$$

be given. Then $f$ is homotopic in $V^{*}-h(L)$ to a mapping $f^{\prime}$ of $\mathrm{Bd} \Delta^{2}$ into $V^{*}-h(K)$. In fact, if $k \leqq n-2$, we can do this with a small homotopy. If $k=n-1$, we must first adjust $f$ slightly so that its image meets $h(K)$ at only a finite number of "piercing" points in $h\left(\right.$ Int $\left.s^{k}\right)$ (see [7, Lemma 5]), and then perform a homotopy in $V^{*}$ which pushes the adjusted image of $f$ over the free edge $h\left(t^{k-1}\right)$. If $k=n$, $n \geqq 3$, we first adjust $f$ slightly (as in the previous case) so as to pierce $h\left(t^{k-1}\right)$ at a finite number of points in $h\left(\right.$ Int $\left.t^{k-1}\right)$ and then perform a homotopy in $V^{*}$ which deforms the image of $f$ away from $h\left(\right.$ Int $\left.s^{k}\right)$. Finally we deform the image completely off $h(K)$ (in each of the two cases where $k \geqq n-1$, we are making use of the fact that each "side" of the complement in Int $M^{n}$ of a topological $(n-1)$-cell is uniformly locally arcwise connected at each point of the $(n-1)$-cell-see Lemma 10 of [7]).

Now $f^{\prime}$ is homotopic to a constant in $U^{*}-h(K)$ and hence $f$ extends to a continuous mapping

$$
F: \Delta^{2} \rightarrow U^{*}-h(L) .
$$

Consider the closed set $Z=F^{-1}(\bar{W})$ in Int $\Delta^{2}$. Since $F \mid Z: Z \rightarrow U-h(L)$ is homotopic to a constant mapping, $F \mid Z$ extends to a mapping $F^{*}: \Delta^{2} \rightarrow U-h(L)$. Define

$$
F \#: \Delta^{2} \rightarrow U-h(L)
$$

by

$$
\begin{aligned}
F \#(x)=F(x), & \text { if } F(x) \in Q \cup \bar{W}, \\
& =F^{*}(x), \quad \text { if } F(x) \in\left(U^{*}-\bar{Q}\right) \cup \bar{W} .
\end{aligned}
$$

It is easily verified that $F \#$ is the required continuous extension of $f$. This completes the proof.

3. Applications to piercing points. I wish to thank R. H. Bing for greatly simplifying my original proof of the following theorem.

TheOREm 2. Let $D$ be a 2-cell topologically embedded in the interior of a 3-manifold $M^{3}$, and let $X \subset$ Int $D$ be cellular in $M^{3}$. Then, with the possible exception of one point, $D$ can be pierced with a tame arc at each point of $X$. 
Proof. By an obvious generalization of Theorem 5 of [3], we may assume without loss of generality that $M^{3}=S^{3}$ and that $D$ is contained in a topological 2-sphere $S \subset S^{3}$. Hence, an arc pierces $D$ at a point $p \in X$ if and only if it pierces $S$ at $p$.

Suppose that there are distinct points $x \in X, y \in X$ such that $D$ can be pierced with a tame arc neither at $x$ nor at $y$. By [10], there is an arc $A \subset$ Int $D$ such that the endpoints of $A$ are $x$ and $y$, and such that $A$ is locally tame at each point of Int $A$. Now $A$ cannot be cellular in $S^{3}$, for if it were, it would be locally tame at one of its endpoints (Lemma 4 of [11]). It would then follow by [6, Theorem 6] that $D$ could be pierced with a tame arc at this endpoint. We shall arrive at a contradiction by showing that $A$ is cellular in $S^{3}$.

Let $D^{*}$ be a 2-cell in $S^{3}$ which contains $A \cup X$ and such that $D^{*}$ is locally polyhedral at each point of $D^{*}-(A \cup X)$ (see [2]). We claim that $D^{*}$ is actually locally tame at each point of $D^{*}-X$. For if $p \in A$ $-X$, then $p$ has a 2-cell neighborhood $N$ in $D^{*}$ such that $N \cap X=\varnothing$, such that $N \cap A$ is a tame spanning arc of $N$ and such that $N$ is locally polyhedral at each point of $N-A$. By [5, Theorem 1], $N$ is tame, and so $D^{*}$ is locally tame at $p$.

Since $D^{*}$ is locally tame at each point of $D^{*}-X$, and since $X$ is cellular in $S^{3}$, it follows that $D^{*}$ is cellular in $S^{3}$. By Theorem $1, A$ is cellular in $S^{3}$. This is the required contradiction.

We now give an application of Theorem $2 .{ }^{2}$

THEOREM 3. Let $S$ be a 2-sphere topologically embedded in $S^{3}$ in such $a$ way that each component of $S^{3}-S$ is an open 3-cell. Then with the possible exception of two points, $S$ can be pierced with a tame arc at each of its points.

Proof. Let $K$ be one of the crumpled cubes bounded by $S$, and let $h$ be a homeomorphism of $K$ into $S^{3}$ such that the closure $C$ of $S^{3}-h(K)$ is a 3-cell (see [8] and [9]). Then $C$ is cellular in $S^{3}$. Further, by Theorem 1 each arc in $\mathrm{Bd} C$ is cellular in $S^{3}$.

Hence by the preceding theorem, at most one point $p$ of $S$ fails to be a piercing point of $K$. Similarly, if $K^{*}$ is the other crumpled cube bounded by $S$, then at most one point $q$ of $S$ fails to be a piercing point of $K^{*}$. By Theorem 4 of [13], $S$ can be pierced with a tame arc at each point of $S-\{p, q\}$.

Although we give no application of the following theorem here, we include it because we consider its proof slightly novel, and because we believe it will be useful in proofs in which one needs to tame a surface "from one side" (in the sense of [8] and [9]).

\footnotetext{
2 Theorem 3 has been obtained independently by L. D. Loveland.
} 
TheOREM 4. Let $K$ be a crumpled cube in $S^{3}$, and let $h: K \rightarrow S^{3}$ be a homeomorphism such that the closure $C$ of $S^{3}-h(K)$ is a 3-cell. Then $h$ preserves the cellularity of subsets of $S=\mathrm{Bd} K$. That is, if $X \subset S$ is cellular in $S^{3}$, then $h(X)$ is cellular in $S^{3}$.

Proof. Let $X \subset S$ be cellular in $S^{3}$. Let $\pi$ be a mapping of $S^{3}$ onto $S^{3}$ whose only inverse set is $X$ (see [4]). That is, $X$ is the only nondegenerate set of the form $\pi^{-1}(y)$. By [8] and [9], there is a homeomorphism $g$ of the crumpled cube $\pi(K)$ in to $S^{3}$ such that the closure $D$ of $S^{3}-g \pi(K)$ is a 3-cell. Now $f=g \pi h^{-1}$ is a mapping of $h(K)$ onto $g \pi(K) \subset S^{3}$ whose only inverse set is $h(X)$. Further, $f \mid \mathrm{Bd} C$ is a monotone mapping of $\mathrm{Bd} C$ onto $\mathrm{Bd} D$. It is not hard to prove that $f \mid \mathrm{Bd} C$ extends to a mapping of the 3-cell $C$ onto the 3-cell $D$ such that $f \mid$ Int $C$ is a homeomorphism (see, for example, $\$ 3$ of [1]). Hence, by piecing together these mappings, we see that there is a mapping of $S^{3}$ onto itself whose only inverse set is $h(X)$, and the result follows.

\section{REFERENCES}

1. R. H. Bing, Some monotone decompositions of a cube, Ann. of Math. 61 (1955), 279-288.

2. - Approximating surfaces with polyhedral ones, Ann. of Math. 65 (1957), $456-483$.

3. - A surface is tame if its complement is 1-ULC, Trans. Amer. Math. Soc. 101 (1961), 294-305.

4. M. Brown, $A$ proof of the generalized Schoenflies theorem, Bull. Amer. Math. Soc. 66 (1960), 74-76.

5. P. H. Doyle and J. G. Hocking, Some results on tame disks and spheres in $E^{3}$, Proc. Amer. Math. Soc. 11 (1960), 832-836.

6. D. S. Gillman, Side approximation, missing an arc, Amer. J. Math. 85 (1963), 459-476.

7. J. P. Hempel and D. R. McMillan, Jr., Locally nice embeddings of manifolds, Amer. J. Math. 88 (1966), 1-19.

8. N. Hosay, The sum of a real cube and a crumpled cube is $S^{3}$, (corrected title), Abstract 607-17, Notices Amer. Math. Soc. 10 (1963), 666.

9. L. L. Lininger, Some results on crumpled cubes, Trans. Amer. Math. Soc. 118 (1965), 534-549.

10. J. Martin, Tame arcs on disks, Proc. Amer. Math. Soc. 16 (1965), 131-133.

11. - The sum of two crumpled cubes, Michigan Math. J. 13 (1966), 147-151.

12. D. R. McMillan, Jr., A criterion for cellularity in a manifold, Ann. of Math. 79 (1964), 327-337.

13. - Some topological properties of piercing points, Pacific J. Math. 22 (1967), 313-322.

14. J. H. C. Whitehead, Simplicial spaces, nuclei and m-groups, Proc. London Math. Soc. 45 (1939), 243-327.

UNIVERSITY OF WISCONSIN 